\title{
Outbreak of febrile illness caused by coxsackievirus A4 in a nursery school in Beijing, China
}

\author{
Jin-Song Li ${ }^{1 \dagger}$, Xiao-Gen Dong ${ }^{2+}$, Meng Qin², Zhi-Ping Xie ${ }^{1}$, Han-Chun Gao ${ }^{1}$, Jun-Yong Yang ${ }^{2}$, Xiao-Xin Yang ${ }^{2}$,
} Dan-Di Li ${ }^{1}$, Jie $\mathrm{Li}^{2^{*}}$ and Zhao-Jun Duan ${ }^{1 *}$

\begin{abstract}
Background: Coxsackievirus A4 (CV-A4) is classified as human enterovirus A according to its serotype. CV-A4, an etiological agent of hand, foot, and mouth disease, affects children worldwide and can circulate in closed environments such as schools and hospitals for long periods.

Findings: An outbreak of febrile illness at a nursery school in Beijing, China, was confirmed to be caused by CV-A4. Phylogenetic analysis of the complete genome of the isolated strain showed that the virus belongs to the same cluster as the predominant CV-A4 strain in China. This outbreak was controlled by effective measures.

Conclusions: The early identification of the pathogen and timely intervention may be the most critical factors in controlling an outbreak caused by CV-A4 in a preschool.
\end{abstract}

Keywords: Coxsackievirus A4, Outbreak, Preschool, Prevention, Control

\section{Findings}

Human enteroviruses (HEVs) are a group of positivesense single-stranded RNA viruses belonging to the genus Enterovirus, in the family Picornaviridae. HEVs are associated with hand, foot, and mouth disease (HFMD), herpangina and even death, and cause both sporadic infections and outbreaks, which often peak in summer and early autumn. Herpangina, predominantly caused by coxsackieviruses, often manifests as high fever, sore throat, and reduced appetite.

The genus Enterovirus includes 12 species. CV-A4 is classified as HEV-A based on its serotype, and is an etiological agent of HFMD [1-3]. The virus can be detected in throat swabs from herpangina patients [4], and can cause severe central nervous system symptoms [5]. $\mathrm{CV}-\mathrm{A} 4$ can circulate in closed environments for long periods, which may be attributable to its prolonged shedding in stools, poor hand hygiene, and/or close

\footnotetext{
*Correspondence: lijie7111@sina.com; zhaojund@126.com

${ }^{\dagger}$ Equal contributors

${ }^{2}$ Fengtai District Center for Disease Control and Prevention of Beijing, 3 Xi An St., Feng-Tai District, Beijing 100071, China

${ }^{1}$ National Institute for Viral Disease Control and Prevention, China CDC, 100 Ying-Xin St., Xuan-Wu District, Beijing 100052, China
}

contact [6]. CV-A4 has also been isolated from drinking water [7] and river water [8].

Here, we identified a febrile illness outbreak associated with CV-A4 in a preschool in China. The school, located in an urban district of Beijing, comprised six classes with 25-30 children in each class. One hundred sixty-five children, aged $<7$ years, were in the care of 40 staff members. The class with the most cases of illness had 27 members, whereas another class with fewer cases had 26 children. "Case" is defined as any individual with symptoms that included a fever, cough, runny nose, and pharyngeal inflammation or a sore throat. "Intimate contact" is defined as any staff member or student with none of these symptoms. "Transmission rate" and "transmission period" are defined as reported previously [6]. The demographic and clinical data for the study subjects were obtained from the school and informed consent was given by the parents/guardians of the subjects.

On 24 May 2011, a 4 year-old boy presenting with a mild fever $\left(38.1{ }^{\circ} \mathrm{C}\right)$ and pharyngeal inflammation was diagnosed with an acute upper-respiratory infection. The number of cases then increased rapidly. By 10 June 2011, 23 children in the preschool showed similar 
symptoms: six were clinically confirmed as suffering an upper-respiratory tract infection and 17 had herpangina. Twenty cases were from Class One and the other three were from a nearby class. Their ages ranged from 3 to 5 years (mean age, 4.3 years) and the sex ratio (male:female) was 18:5 (3.6:1). Most of the children displayed a self-limiting illness, lasting for 2-4 days. All the patients had a good prognosis and there were no fatalities during this outbreak.
The most common symptoms in the affected children were fever (100 \%; range, $\left.37.9-41.0{ }^{\circ} \mathrm{C}\right)$ and pharyngeal inflammation or sore throat $(21 / 23,91.3 \%)$. One boy had a cough and three had runny noses. No other symptoms were noted in any of the subjects. The mean duration of fever was 2.70 days (95\% confidence interval, 2.34-3.05). In seven cases, the peak body temperature was $>38.9{ }^{\circ} \mathrm{C}$. Leukocytosis (white blood cell count $>$ $15 \times 10^{9} / \mathrm{L}$ ) was noted in four cases (Table 1$)$. The peak

Table 1 The symptoms observed for each of the cases during the outbreak and the pathogens isolated

\begin{tabular}{|c|c|c|c|c|c|c|c|c|c|}
\hline No. & Sample name & $\begin{array}{l}\text { Date of } \\
\text { sample }\end{array}$ & Temperature $\left({ }^{\circ} \mathrm{C}\right)$ & Cough & $\begin{array}{l}\text { Pharyngeal inflammation } \\
\text { or sore throat }\end{array}$ & $\begin{array}{l}\text { Nasal } \\
\text { discharge }\end{array}$ & WBC & Virus isolation & CPE \\
\hline 1 & case 1 & $6 / 1 / 2011$ & 39 & $\mathrm{~N}$ & Y & $N$ & WBC $15 \times 10^{9} / \mathrm{L}$ & $\mathrm{NE}$ & UN \\
\hline 2 & case2 & $6 / 1 / 2011$ & 38.6 & $\mathrm{~N}$ & Y & $N$ & UN & CV-A4 & $P$ \\
\hline 3 & case3 & $6 / 1 / 2011$ & 38.5 & $\mathrm{~N}$ & Y & $N$ & UN & CV-A4 & $\mathrm{NE}$ \\
\hline 4 & case4 & $6 / 1 / 2011$ & 38.1 & $\mathrm{~N}$ & Y & N & UN & CV-A4 & $\mathrm{NE}$ \\
\hline 5 & case5 & $6 / 1 / 2011$ & 41 & $\mathrm{~N}$ & Y & $N$ & UN & CV-A4 & $P$ \\
\hline 6 & case6 & $6 / 1 / 2011$ & 39.4 & $\mathrm{~N}$ & Y & $N$ & UN & CV-A4, HAdV-3 & $P$ \\
\hline 7 & case7 & $6 / 1 / 2011$ & 39.4 & $\mathrm{~N}$ & $N$ & Y & WBC $12 \times 10^{9} / \mathrm{L}$ & CV-A4 & $\mathrm{P}$ \\
\hline 8 & case8 & $6 / 1 / 2011$ & 38.5 & $\mathrm{~N}$ & Y & $N$ & WBC $12.6 \times 10^{9} / L$ & Influenza B & UN \\
\hline 9 & case9 & $6 / 1 / 2011$ & 38 & $\mathrm{~N}$ & Y & Y & UN & CV-A4 & $P$ \\
\hline 10 & case10 & $6 / 1 / 2011$ & 37.9 & $\mathrm{~N}$ & Y & $N$ & UN & CV-A4 & $P$ \\
\hline 11 & case11 & $6 / 1 / 2011$ & 37.9 & $\mathrm{~N}$ & Y & Y & UN & CV-A4 & $P$ \\
\hline 12 & case12 & $6 / 1 / 2011$ & 39.8 & $\mathrm{~N}$ & Y & $N$ & UN & CV-A4 & $P$ \\
\hline 13 & case13 & $6 / 3 / 2011$ & 39 & Y & Y & $\mathrm{N}$ & WBC $17 \times 10^{9} / \mathrm{L}$ & CV-A4 & $\mathrm{P}$ \\
\hline 14 & case14 & $6 / 3 / 2011$ & 38 & $\mathrm{~N}$ & Y & $N$ & UN & CV-A4 & $P$ \\
\hline 15 & case15 & $6 / 3 / 2011$ & 38.8 & $\mathrm{~N}$ & Y & $N$ & WBC $15 \times 10^{9} / L$ & CV-A4 & NE \\
\hline 16 & case16 & $6 / 3 / 2011$ & 39 & N & Y & $N$ & UN & CV-A4 & $P$ \\
\hline 17 & case17 & $6 / 3 / 2011$ & 39 & $\mathrm{~N}$ & Y & $N$ & WBC $17.8 \times 10^{9} / \mathrm{L}$ & NE(bacteria positive) & UN \\
\hline 21 & Intimate contact1 & $6 / 8 / 2011$ & normal & / & / & / & UN & $\mathrm{NE}$ & UN \\
\hline 23 & Intimate contact2 & $6 / 8 / 2011$ & normal & / & / & / & UN & $\mathrm{NE}$ & UN \\
\hline 24 & Intimate contact3 & $6 / 8 / 2011$ & normal & / & / & / & UN & $\mathrm{NE}$ & UN \\
\hline 25 & Intimate contact4 & $6 / 8 / 2011$ & normal & / & / & / & UN & NE & UN \\
\hline 26 & Intimate contact5 & $6 / 8 / 2011$ & normal & / & / & / & UN & NE & UN \\
\hline 27 & Intimate contact6 & $6 / 8 / 2011$ & normal & / & / & / & UN & HRV-C,HAdV-3,CV-A4 & $P$ \\
\hline 28 & Intimate contact7 & $6 / 8 / 2011$ & normal & / & / & / & UN & $\mathrm{NE}$ & UN \\
\hline 29 & case18 & $6 / 8 / 2011$ & 38.1 & $\mathrm{~N}$ & Y & $N$ & UN & HRV-A & $\mathrm{NE}$ \\
\hline 30 & case19 & $6 / 8 / 2011$ & 39.2 & $\mathrm{~N}$ & $N$ & N & UN & $\mathrm{CV}-\mathrm{A} 4, \mathrm{HAdV}-3$ & $\mathrm{NE}$ \\
\hline 31 & case20 & $6 / 8 / 2011$ & 38.1 & $\mathrm{~N}$ & Y & $N$ & UN & CV-A4 & NE \\
\hline 32 & case21 & $6 / 8 / 2011$ & 38 & $\mathrm{~N}$ & Y & $N$ & UN & CV-A4 & NE \\
\hline 33 & Intimate contact8 & 6/8/2011 & normal & / & / & / & UN & CV-A4,HRV-A & $\mathrm{NE}$ \\
\hline 34 & Intimate contact 9 & $6 / 8 / 2011$ & normal & / & / & / & UN & CV-A4,HRV-A & $\mathrm{NE}$ \\
\hline 35 & Intimate contact 10 & $6 / 8 / 2011$ & normal & / & / & / & UN & $\mathrm{NE}$ & UN \\
\hline 36 & Intimate contact 11 & $6 / 8 / 2011$ & normal & / & / & / & UN & CV-A4,HRV-A & NE \\
\hline 37 & case 22 & no sample & 38.3 & $\mathrm{~N}$ & Y & $N$ & UN & UN & UN \\
\hline 38 & case23 & no sample & 37.9 & $\mathrm{~N}$ & Y & $N$ & UN & UN & UN \\
\hline
\end{tabular}

$N$, no symptoms; $Y$, symptoms observed; /, none of the symptoms observed; UN, undetected; $N E$, no viruses isolated from samples or cells; $P$, CV-A4 was isolated from cells; WBC, white blood cell; $C V-A 4$, coxsackievirus A4; HAdV-3, human adenovirus 3; $H R V-A$, human rhinovirus A; $C P E$, cytopathic effect 


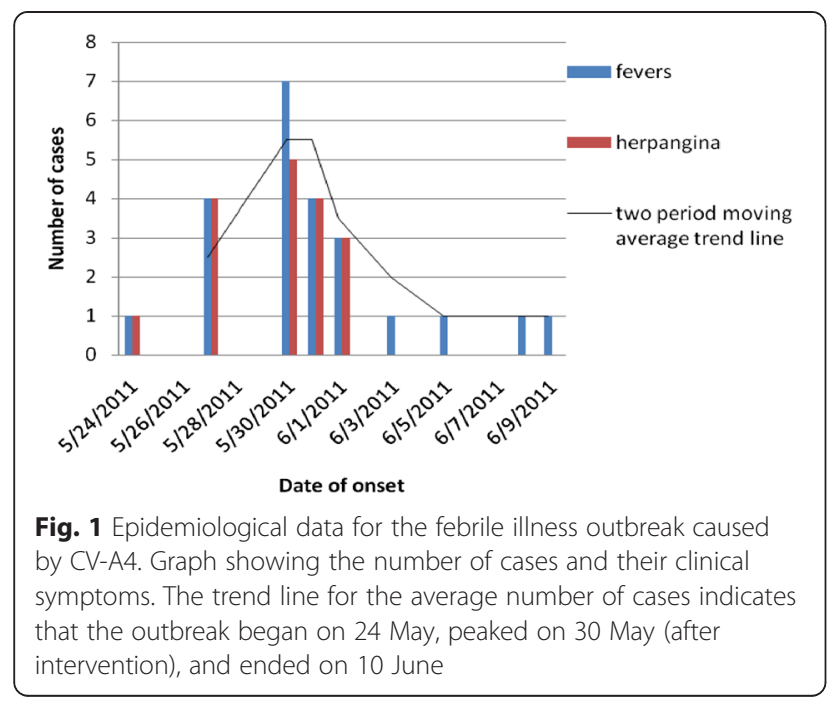

incidence was between 27 May and 1 June, accounting for $78.3 \%$ (18/23) of cases (Fig. 1).

Thirty-two oropharyngeal swabs were taken from 21 patients (no samples were available for two cases) and 11 intimate contacts from the two classes. All 32 swab specimens were stored in virus preservation solution at $-70{ }^{\circ} \mathrm{C}$ until analysis. The nucleic acids were extracted with a QIAamp MinElute Virus Spin Kit (Qiagen, Valencia, CA, USA), according to the manufacturer's instructions. All the samples were screened by PCR or RT-PCR for influenza A and B viruses, respiratory syncytial virus, human rhinovirus, human echovirus, parainfluenza viruses $1,2,3$ and 4, human metapneumovirus, human coronavirus NL63, human adenovirus, and some HEVs, as reported previously [9-13]. The positive PCR products were purified and sequenced.

Of the 32 oropharyngeal swabs collected, 21 were positive for CV-A4. The detection rates in the patients and their intimate contacts were $81.0 \%(17 / 21)$ and $36.4 \%(4 / 11)$, respectively. Human adenovirus-3, human rhinovirus, and influenza $B$ were also detected, but the samples were negative for the other viruses tested (Table 1). Outbreaks of human coxsackievirus infections in preschools are reported frequently worldwide and are typically associated with HFMD caused by the coxsackie A16 virus [14]. However, febrile outbreaks caused by $\mathrm{CV}-\mathrm{A} 4$ are rarely reported in preschools. In this study, 17 of the 21 tested cases were CV-A4 positive, strongly suggesting that this outbreak was caused by CV-A4.

Herpangina diseases are asymptomatic or self-limiting infections, often caused by EV71 or coxsackieviruses, including CV-A4. Chia-Jie Lee reported that herpangina caused by CV-A4 usually presented with high fever $>$ $39.0{ }^{\circ} \mathrm{C}$ (75.5\%), oral ulcers (80.6\%), and leukocytosis
(34.0\%), and that fever lasted longer than 3 days in $23.5 \%$ of sufferers [15]. In another outbreak in a preschool class, the transmission rate was $25.9 \%(7 / 27)$ and the transmission period was 28 days [6]. In our study, $42.9 \%(9 / 21)$ of cases had high fever, and the fever persisted for more than 3 days in $14.3 \%$ of patients $(3 / 21)$ and for 3 days in $42.9 \%$ of patients $(9 / 21)$. The transmission rate was $74.1 \%(20 / 27)$ in Class One, the transmission period was 18 days, and two CV-A4positive patients had leukocytosis (leukocyte counts $\geq$ $15,000 / \mu \mathrm{L}$ ). We did not measure the white blood cell counts in all cases.

The virus was isolated from all CV-A4-positive throat samples in human rhabdomyosarcoma cells, using a previously reported protocol [16]. Cytopathic effects were observed in $52.2 \%(12 / 21)$ of the samples incubated with rhabdomyosarcoma cells. Twelve VP1 sequences and three complete genomes of CV-A4 (two from initial cases and one from intimate contact FT27) were amplified from the cell culture supernatants by RT-PCR and rapid amplification of cDNA ends-PCR, according to the manual supplied with the SMARTer ${ }^{\text {Tm }}$ RACE cDNA Amplification Kit (Japan, Tokyo) [17]. The primer sequences and details of the reaction conditions are given in Table 2. The genome of CV-A4 is about $7460 \mathrm{bp}$ and the VP1-encoding fragment is $915 \mathrm{bp}$. The genomes of three strains (FT27, FT7, and FT5) were 98.5-99.5\% homologous and the VP1 fragments of nucleic acids were 99.9-100\% homologous. These sequences shared 95.5-96.5 \% identity with the clinical CV-A4 strain previously isolated in mainland China (GenBank: HQ728260).

Table 2 Primers used to amplify the complete CV-A4 genome

\begin{tabular}{lll}
\hline Primer name & Primer sequence & Fragment (bp) \\
\hline F1 & TTAAAACAGCCTGTGGGTTG & $\sim 300$ \\
R1 & AACCCATAGGCAGGCCGCC & \\
cox241 & ACCCGGCTAACTACTTCGAGAAC & 1853 \\
cox2093 & ATGAATGAGCCCGTAAACATGAAA & \\
M1908 & AGGTGTAAGCCGGTTGCTCATAC & 1025 \\
M2932 & CACTGGAACGATTCTCGAGCATC & \\
VP1A(2319) & CTTCGTAGTGCCACCAGACAC & 1066 \\
VP1S(3384) & AGCTCCAGATTGTTGACCGA & \\
N2846 & TCACCTTCGTCACCAATCTAG & 1012 \\
N3857 & CCTGGAGACTGCGTCAGTGA & \\
bcox3615 & CAGTGAGTACTACCCTGCCAGGTATCAA & 1318 \\
bcox4932 & TGCGGTGTATTTCAGAGCACAGTTG & \\
ccox4610 & AGCAAGTGGTCACTGTCATGGATGA & 1324 \\
ccox5933 & CCTGCACAAAAGCCCTGCCTGCCAT & \\
dcox5856 & TGGAGGAGTAGTACATCAGTTGA & 1548 \\
dcox7403 & CAGTTATGTTCACGACCAGATTCT & \\
ecox-a(race pcr 3') & ATGCCAATGAAGGAGATCATGAGTCC & $\sim 300$ \\
\hline
\end{tabular}


As expected, the polyproteins of FT7 and the CV-A4 mainland strain (GenBank: HQ728260) shared $96.5 \%$ identity (VP4, $97.1 \%$; VP2, $96.7 \%$; VP3, $96.5 \%$; and VP1, $96.2 \%$ ) and the nonstructural proteins shared $95.8 \%$ identity (2A, $95.1 \%$; 2B, $98.3 \%$; 2C, $95.3 \%$; 3A, $94.0 \%$; 3B, $98.5 \%$; 3C, $96.7 \%$; and 3D, $96.2 \%$ ).

In a phylogenetic analysis of the near-complete genome of the CV-A4 strain isolated in this outbreak, it clustered with the only two complete genomes of CVA4 deposited in the GenBank database (Fig. 2). Analysis of a 3' partial VP1 sequence, showed all CV-A4 isolates from this outbreak clustered on the same branch and were closely related to the predominant $\mathrm{CV}$-A4 strains JL/CHN/2006 (GenBank: JQ715709) and SD/CHN/2008 (GenBank: GQ253374). All CV-A4 isolates from mainland China, Japan, India, and Taiwan, including CV-A4-mainland (GenBank: HQ728260), formed a subgenogroup, and isolates from the USA formed another subgenogroup within genotype III. Global $\mathrm{CV}-\mathrm{A} 4$ strains show regional variations and were classed into three genotypes based on their $3^{\prime}$ partial VP1 sequences $[3,18]$. Our results indicate that the
CV-A4 strain isolated in this study probably also varies across its geographic distribution (Fig. 2). This $\mathrm{CV}-\mathrm{A} 4$ strain is the predominant strain in China, resulting from the rapid lineage turnover of $\mathrm{CV}-\mathrm{A} 4$ and the replacement of previously circulating strains by this new dominant variant [18].

CV-A4 is primarily transmitted via the fecal-oral route by contaminated hands, toys, and food. When the local Center for Disease Control received the report of this outbreak on June 1, normal control measures were taken, such as hand-washing, disinfection of the classroom, and body temperature screening. Health education was intensified and the public activities at all of the nursery schools were cancelled, as described previously $[19,20]$. On June 1 , the children in Classes One and Two celebrated Children's Day with a party. On June 3, when the pathogens causing this outbreak were identified, the local Center for Disease Control suspended Class One for 10 days. During the outbreak, 20 children in Class One and three children in Class Two caught the disease. Although the transmission period of CV-A4 within a class is reported to be 28 days (6), this outbreak
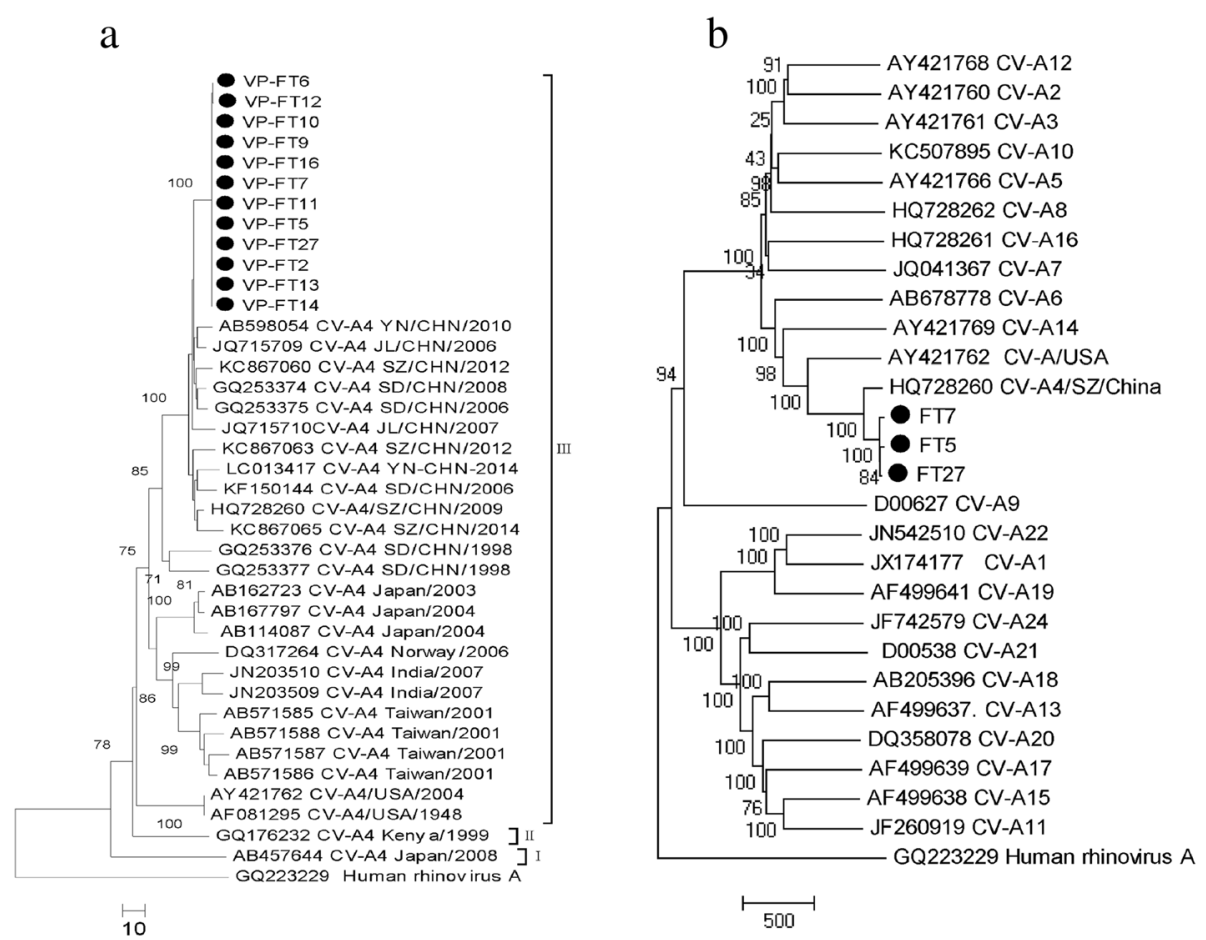

Fig. 2 Phylogenetic analysis of the pathogens isolated during the outbreak. A phylogenetic analysis of the nucleotide sequences of the viruses isolated during the outbreak: (a) the three partial VP1 sequences of CVA-4; (b) the near-complete nucleotide sequences of strains of human Coxsackie A virus. Phylogenetic analysis was performed and the tree was constructed using the neighbor-joining algorithm implemented in the MEGA version 5.0 software with 1000 bootstrap pseudoreplicates. The numbers on the branches indicate the bootstrap values, excluding those of $<70 \%$ for clarity. Human rhinoviruses (HRVs) were used as outgroups on both trees. Red dots show the CV-A4 strains isolated in this study and the black dots show the only complete genome of CV-A4 in the GenBank database 
was controlled within 18 days. All the control measures used in this outbreak were similar to those used for HMFD caused by EV71.

\section{Conclusions}

This outbreak of febrile illness at a preschool in Beijing, China, was caused by CV-A4. Measures, such as surveillance in peak season, early identification of the pathogen and timely intervention, are key to controlling outbreaks of disease caused by CV-A4 or other HEVs.

\section{Nucleotide sequence accession numbers}

The three complete genomes have been submitted to the GenBank database under accession numbers KP676984, KP676985, and KP676986. Partial sequences of CV-A4 and other viral sequences have been deposited under accession numbers KP676948-KP676983.

\section{Abbreviations \\ CV-A4: Coxsackievirus A4; HEV: Human enterovirus; HFMD: Hand, foot, and mouth disease.}

\section{Competing interests}

The authors declare that they have no competing interests.

\section{Authors' contributions}

JSL identified the pathogens causing this outbreak, performed the genetic analysis, and drafted the manuscript; XGD collected the samples and undertook the epidemiological investigation; MQ collected the samples and undertook the epidemiological investigation; ZPX identified the pathogens causing the outbreak; GHC isolated the viruses and participated in the whole-genome sequencing; DDL participated in the whole-genome sequencing; JYY conducted the epidemiological investigation and outbreak control; XXY conducted the epidemiological investigation and outbreak control; JL participated in the design of the study; and ZJD participated in the design of the study and edited the manuscript. All authors have read and approved the final manuscript.

\section{Acknowledgments}

We thank all the staff members of the preschool who helped with this study.

Received: 31 January 2015 Accepted: 15 June 2015

Published online: 18 June 2015

\section{References}

1. Witso E, Palacios G, Cinek O, Stene LC, Grinde B, Janowitz D, et al. High prevalence of human enterovirus a infections in natural circulation of human enteroviruses. J Clin Microbiol. 2006:44:4095-100.

2. Yang F, Zhang T, Hu Y, Wang X, Du J, Li Y, et al. Survey of enterovirus infections from hand, foot and mouth disease outbreak in China, 2009. Virol J. 2011:8:508.

3. Hu YF, Yang F, Du J, Dong J, Zhang T, Wu ZQ, et al. Complete genome analysis of coxsackievirus $\mathrm{A} 2, \mathrm{~A} 4, \mathrm{~A} 5$, and $\mathrm{A} 10$ strains isolated from hand, foot, and mouth disease patients in China revealing frequent recombination of human enterovirus A. J Clin Microbiol. 2011;49:2426-34.

4. Zavate O, Avram G, Pavlov E, Burlea-Iriciuc A, Ivan A, Cotor F. Coxsackie A virus-associated herpetiform angina. Virologie. 1984;35:49-53.

5. Carey DE, Myers RM. Isolation of type A4 coxsackie virus from the blood serum of a child with rapidly fatal illness marked by severe central nervous system manifestations. Indian J Med Res. 1969;57:765-9.

6. Wu PC, Huang LM, Kao CL, Fan TY, Cheng AL, Chang LY. An outbreak of coxsackievirus A16 infection: comparison with other enteroviruses in a preschool in Taipei. J Microbiol Immunol Infect. 2010;43:271-7.

7. Nestor I, Costin L, Sovrea D, lonescu N. Investigations on the presence of enteroviruses in drinking water. Virologie. 1978;29:203-7.
8. Simkova A, Wallnerova Z. Survival of small amounts of Coxsackie A4 virus in Danube river water under laboratory conditions. Acta Virol. 1973;17:505-6.

9. Lu X, Erdman DD. Molecular typing of human adenoviruses by PCR and sequencing of a partial region of the hexon gene. Arch Virol. 2006;151:1587-602.

10. Bellau-Pujol S, Vabret A, Legrand L, Dina J, Gouarin S, PetitjeanLecherbonnier J, et al. Development of three multiplex RT-PCR assays for the detection of 12 respiratory RNA viruses. J Virol Methods. 2005;126:53-63.

11. Arthur JL, Higgins GD, Davidson GP, Givney RC, Ratcliff RM. A novel bocavirus associated with acute gastroenteritis in Australian children. PLoS Pathog. 2009;5:e1000391.

12. Donofrio JC, Coonrod JD, Davidson JN, Betts RF. Detection of influenza A and $B$ in respiratory secretions with the polymerase chain reaction. PCR Methods Appl. 1992;1:263-8.

13. Savolainen C, Mulders MN, Hovi T. Phylogenetic analysis of rhinovirus isolates collected during successive epidemic seasons. Virus Res. 2002:85:41-6.

14. Ferson MJ, Bell SM. Outbreak of Coxsackievirus A16 hand, foot, and mouth disease in a child day-care center. Am J Public Health. 1991;81:1675-6.

15. Lee CJ, Huang YC, Yang S, Tsao KC, Chen CJ, Hsieh YC, et al. Clinical features of coxsackievirus A4, B3 and B4 infections in children. PLoS One. 2014;9:e87391

16. Tao Z, Wang H, Li Y, Liu G, Xu A, Lin X, et al. Molecular epidemiology of human enterovirus associated with aseptic meningitis in Shandong Province, China, 2006-2012. PLoS One. 2014;9:e89766.

17. Freeman LA. Cloning full-length transcripts and transcript variants using $5^{\prime}$ and 3' RACE. Methods Mol Biol. 2013;1027:3-17.

18. Chu PY, Lu PL, Tsai YL, Hsi E, Yao CY, Chen YH, et al. Spatiotemporal phylogenetic analysis and molecular characterization of coxsackievirus A4. Infect Genet Evol. 2011;11:1426-35.

19. Solomon T, Lewthwaite P, Perera D, Cardosa MJ, McMinn P, Ooi MH. Virology, epidemiology, pathogenesis, and control of enterovirus 71. Lancet Infect Dis. 2010;10:778-90.

20. McMinn PC. An overview of the evolution of enterovirus 71 and its clinical and public health significance. FEMS Microbiol Rev. 2002;26:91-107.

\section{Submit your next manuscript to BioMed Central and take full advantage of:}

- Convenient online submission

- Thorough peer review

- No space constraints or color figure charges

- Immediate publication on acceptance

- Inclusion in PubMed, CAS, Scopus and Google Scholar

- Research which is freely available for redistribution 